\title{
PATH INTEGRALS FOR PARASTATISTICS
}

\author{
O.W. Greenberg ${ }^{1}$ and A.K. Mishra ${ }^{2}$ \\ Center for Theoretical Physics \\ Department of Physics \\ University of Maryland \\ College Park, MD 20742-4111 \\ University of Maryland Preprint PP 04-044
}

\begin{abstract}
We demonstrate that parastatistics can be quantized using path integrals by calculating the generating functionals for time-ordered products of both free and interacting parabose and parafermi fields in terms of path integrals. We also give a convenient form of the commutation relations for the Green components of the parabose and parafermi operators in both the canonical and path integral formalisms.
\end{abstract}

\section{Introduction}

Canonical quantization [1, 2] and path integral quantization [3, 4] are two widely used methods to quantize a physical system. The generality of these two methods and their equivalence or non-equivalence has general interest. Many physicists, including the present authors, believe that any system that can be quantized using one of these methods also can be quantized using the other. We acknowledge that for technical reasons one or the other method may be simpler or more convenient for any specific system.

Some years ago M.G.G. Laidlaw and C. M. DeWitt [5] in a well-cited influential paper studied path integral quantization for a system of identical particles. They

\footnotetext{
${ }^{1}$ email address, owgreen@physics.umd.edu.

${ }^{2}$ Permanent address Institute of Mathematical Sciences, Chennai 600 113, India, email address: mishra@imsc.res.in
} 
noted the important distinction between quantization of identical particles in two space dimensions and in three or more dimensions. This distinction holds for both path integral and canonical quantization. We believe that their paper correctly proves that parastatistics cannot be based on the topology of three dimensional space (in contrast to anyon statistics [6,7], which is based on the topology of two dimensional space). Although the text of their paper does not mention parastatistics, their abstract contains the phrase "... showing that the Feynman formalism excludes parastatistics." Dr. Cecille DeWitt has informed us that the abstract is misleading and that M. Laidlaw and C. DeWitt did not intend to state that there is a contradiction between path integration and parastatistics [8]. If, indeed, parastatistics fields [9] could not be quantized using path integrals that would have significance for the general question of the equivalence of the canonical and path integral methods of quantization. Our belief that any field theory that can be quantized using the canonical formalism also can be quantized using path integrals was our initial motivation to formulate field theories with parastatistics fields using path integrals.

There are several plausible assumptions about the quantum mechanics of indistiguishable particles that lead directly to the conclusion that only bosons or fermions can exist in three or more space dimensions. These plausible assumptions lead to the same conclusion in the context of path integrals. The basic principles of quantum mechanics do not require these plausible assumptions; indeed without these assumptions quantum mechanics allows the description of indistinguishable particles that have more general statistics than bose or fermi statistics $[10,11]$. Here are some of the plausible assumptions together with the reasons why these plausible assumptions are not required for quantum mechanics: (1) Transposing indistinguishable particles in a quantum state produces a state that is linearly dependent on the initial state, i.e. $T \Psi=\eta \Psi$, where $T$ is a transposition. Since the square of a transposition is the identity this leads directly to $T^{2} \Psi=\eta^{2} \Psi=\Psi$, so $\eta= \pm 1$. In a general representation of the symmetric group this is not the case. Greenberg and Messiah showed [10] that quantum mechanics can be formulated for an arbitrary represention of the symmetric group. (2) The space of quantum states obtained by permuting indistiguishable particles is coherent, i.e., these states can be superposed to form other states without restriction. For states of several indis- 
tinguishable particles there is an absolute selection rule that prohibits transitions between states in inequivalent irreducible representations of the symmetric group so that the relative phases of states in inequivalent irreducible representations can never be measured. Such systems must be treated as statistical mixtures rather than as coherent superpositions of states. In short, there is a superselection rule that separates states in inequivalent irreducible representations of the symmetric group [10, 12]. If one assumes all states can be superposed, then since there is a symmetric state and an antisymmetric state of $N$ particles for each $N$, only bosons and fermions can occur. (3) The configuration space of a system of particles is in one-to-one correspondence with the states of the system. This assumption implies that the states always can be superposed and leads back to assumption (2). (4) The homotopy classes of the configuration space determine the particle statistics. The discussion of irreducible representations of the symmetric group shows that the existence of different homotopy classes allows different types of statistics, but there is no requirement that each type of statistics must be associated with a different homotopy class of the configuration space.

\section{Canonical Quantization of Parastatistics}

H.S. Green [9] proposed the first proper quantum statistical generalization of bose and fermi statistics. Green noticed that the commutator of the number operator with the annihilation and creation operators is the same for both bosons and fermions

$$
\left[n_{k}, a_{l}^{\dagger}\right]_{-}=\delta_{k l} a_{l}^{\dagger}
$$

The number operator can be written

$$
n_{k}=(1 / 2)\left[a_{k}^{\dagger}, a_{k}\right]_{ \pm}+\text {const. }
$$

where the anticommutator $\left[a_{k}^{\dagger}, a_{k}\right]_{+}$(commutator $\left.\left[a_{k}^{\dagger}, a_{k}\right]_{-}\right)$is for the bose (fermi) case. If these expressions are inserted in the number operator-creation operator commutation relation, the resulting relation is trilinear in the annihilation and creation operators. Polarizing the number operator to get the transition operator $n_{k l}$ which annihilates a free particle in state $l$ and creates one in state $k$ leads to Green's 
trilinear commutation relation for his parabose and parafermi statistics,

$$
\left[\left[a_{k}^{\dagger}, a_{l}\right]_{ \pm}, a_{m}^{\dagger}\right]_{-}=2 \delta_{l m} a_{k}^{\dagger}
$$

Since these rules are trilinear, the usual vacuum condition,

$$
a_{k}|0\rangle=0
$$

does not suffice to allow calculation of matrix elements of the $a^{\prime}$ 's and $a^{\dagger}$ 's; a condition on single-particle states must be added,

$$
a_{k} a_{l}^{\dagger}|0\rangle=p \delta_{k l}|0\rangle
$$

Green found an infinite set of solutions of his commutation rules, one for each positive integer $p$, by giving an ansatz which he expressed in terms of bose and fermi operators. Let the annihilation and creation operators be represented by a sum of operators with an additional index, the "Green" index,

$$
a_{k}^{\dagger}=\sum_{\alpha=1}^{p} b_{k}^{(\alpha) \dagger}, \quad a_{k}=\sum_{\alpha=1}^{p} b_{k}^{(\alpha)}
$$

and let the $b_{k}^{(\alpha)}$ and $b_{k}^{(\beta) \dagger}$ be bose (fermi) operators for $\alpha=\beta$ but anticommute (commute) for $\alpha \neq \beta$ for the "parabose" ("parafermi") cases. This ansatz clearly satisfies Green's relation since the (polarized) number operator is diagonal in the Green index for each case. The integer $p$ is the order of the parastatistics. The physical interpretation of $p$ is that, for parabosons, $p$ is the maximum number of particles that can occupy an antisymmetric state, while for parafermions, $p$ is the maximum number of particles that can occupy a symmetric state (in particular, the maximum number which can occupy the same state). The case $p=1$ corresponds to the usual bose or fermi statistics. Later, Greenberg and Messiah [10] proved that Green's ansatz gives all Fock-like solutions of Green's commutation rules.

We give a useful form of the commutation relations for the Green components of a parabose (parafermi) operator,

$$
b_{k}^{(\alpha)} b_{l}^{(\beta) \dagger}= \pm\left(2 \delta_{\alpha, \beta}-1\right) b_{l}^{(\beta) \dagger} b_{k}^{(\alpha)}+\delta_{\alpha, \beta} \delta_{k, l},
$$


where the upper (lower) sign is for parabose (parafermi); analogous forms hold when both operators are annihilation or both are creation operators, but the $\delta_{\alpha, \beta} \delta_{k, l}$ term is absent. As far as we know this form does not appear in the literature. We find that the $\pm\left(2 \delta_{\alpha, \beta}-1\right)$ factors are very useful in doing systematic calculations.

We discussed quantization of parastatistics above in terms of the commutation relations for parabose and parafermi creation and annihilation operators. Quantization of parastatistics in terms of spacetime-dependent field operators follows for free fields or fields in the interaction picture by the usual Fourier transformations using, for example, plane waves. Thus for a neutral scalar parabose field,

$$
\phi(x)=\frac{1}{(2 \pi)^{3 / 2}} \int \frac{d^{3} k}{\sqrt{2 \omega_{k}}}\left(a_{k} e^{-i k \cdot x}+a_{k}^{\dagger} e^{i k \cdot x}\right), \omega_{k}=\sqrt{\mathbf{k}^{2}+m^{2}} .
$$

In terms of fields, local observables have a form analogous to the usual ones; for example, the local current for a spin-1/2 parafermi theory is

$$
j_{\mu}=(1 / 2)\left[\bar{\psi}(x), \gamma_{\mu} \psi(x)\right]_{-}=\sum_{\alpha=1}^{p} \bar{\psi}^{(\alpha)}(x) \gamma_{\mu} \psi^{(\alpha)}(x)
$$

From Green's ansatz, it is clear that the squares of all norms of states are positive, since sums of bose or fermi operators give positive norms. Thus parastatistics gives a set of orthodox theories.

We give the form of the field commutation relations for parabose and parafermi fields in terms of both the the fields and the Green components of the fields,

$$
\begin{gathered}
{\left[\left[\phi\left(x_{1}\right), \phi\left(x_{2}\right)\right]_{ \pm}, \phi\left(x_{3}\right)\right]_{-}=2 i \Delta\left(x_{2}-x_{3}\right) \phi\left(x_{1}\right) \pm 2 \phi\left(x_{2}\right) i \Delta\left(x_{1}-x_{3}\right)} \\
\phi^{(\alpha)}\left(x_{1}\right) \phi^{(\beta)}\left(x_{2}\right)=\left(2 \delta_{\alpha, \beta}-1\right) \phi^{(\beta)}\left(x_{2}\right) \phi^{(\alpha)}\left(x_{1}\right)+\delta_{\alpha, \beta} i \Delta\left(x_{1}-x_{2}\right)
\end{gathered}
$$

For charged fields, keep only the contractions between a field and its adjoint.

\section{Green Ansatz for Path Integrals}

\subsection{Parabose case}

Since parafields can be constructed in second quantized form using the Green ansatz [9], we have constructed the representations in terms of path integrals by using construc- 
tions closely analogous to those of Green. Since, as mentioned above, Greenberg and Messiah [13] proved that any field theory that obeys the trilinear commutation relation of Green that has a Fock-like representation can be represented using the Green ansatz, the Green ansatz for the path integral can be used without loss of generality.

We recall the generating a (bose) charged scalar field given in terms of a path integral,

$$
\begin{aligned}
& W\left[J, J^{\star}\right]= \\
& \quad \int \mathcal{D} \phi^{\star} \mathcal{D} \phi \exp \left[i \int d^{4} x\left(\mathcal{L}\left(\phi(x), \phi^{\star}(x)\right)+J^{\star}(x) \phi(x)+\phi^{\star}(x) J(x)\right)\right]
\end{aligned}
$$

with

$$
\mathcal{L}=\mathcal{L}_{0}+\mathcal{L}_{I}, \mathcal{L}_{0}=\partial_{\mu} \phi^{\star} \partial^{\mu} \phi-m^{2} \phi^{\star} \phi, \mathcal{L}_{I}=V\left(\phi, \phi^{\star}\right)
$$

Since one can calculate the path integral for the free term $\mathcal{L}_{0}$ explicitly, one often represents the interaction term $\mathcal{L}_{I}$ by a functional derivative. Then

$$
\begin{aligned}
W\left[J, J^{\star}\right]= & W[0,0] \exp \left[i \int d^{4} x V\left(-i \frac{\delta}{\delta J^{\star}(x)},-i \frac{\delta}{\delta J(x)}\right)\right] \\
& \times \exp \left[-i \int d^{4} x d^{4} y J^{\star}(x) \Delta_{F}(x-y) J(y)\right]
\end{aligned}
$$

where $\Delta_{F}(x)=(2 \pi)^{-4} \int d^{4} k \exp (-i k \cdot x)\left(k^{2}-m^{2}-i \epsilon\right)^{-1}$.

We construct the generating functional for time ordered products for parabose fields in parallel with the above construction for bose fields. We must take into account the requirement that the terms allowed in the parabose (and also in the parafermi) Lagrangian must obey local commutativity [13, 14]. These papers show that the para fields must occur in one of two forms. The form $\left[\phi^{\star}(x), \phi(x)\right]_{ \pm}$for any order $p$ of parastatistics is analogous to the requirement that charged fields occur in the combination $\phi^{\star}(x) \phi(x)$ in order to obey a $U(1)$ symmetry. The form of a nested set of $p-1$ commutators and anticommutators for para fields of order $p$ is analogous to the possibility of a term $\epsilon^{\alpha, \beta, \cdots, \zeta} \phi_{\alpha}(x) \phi_{\beta}(x) \cdots \phi_{\zeta}(x)$ in a theory that obeys $S U(n)$ symmetry. We refer to the papers just cited for a demonstration and detailed analysis of these possibilities. In the first case the admissible terms in the Lagrangian will be functions of terms diagonal in the Green index. In the second case the admissible terms will be functions of terms antisymmetric in the Green 
index; that is for a given value of $p$ each index from 1 to $p$ will occur exactly once in each term.

The Lagrangian density for a free parabose field is

$$
\mathcal{L}\left(\phi, \phi^{\star}\right)=\frac{1}{2}\left(\left[\partial_{\mu} \phi^{\star}(x), \partial^{\mu} \phi(x)\right]_{+}-m^{2}\left[\phi^{\star}(x), \phi(x)\right]_{+}\right) .
$$

Using the Green ansatz, the parabose field is represented by

$$
\phi(x)=\sum_{\alpha=1}^{p} \phi^{(\alpha)}(x)
$$

that is, as a sum of $p$ bose fields ( $p$ is the order of the parastatistics). The bose fields with different Green index anticommute rather than commute. In order to obtain the generating functional, we introduce parabose Grassmann fields $J(x)$ [15]. The parabose Grassmann fields have a representation similar to that for the parabose fields, except that the right-hand sides always vanish,

$$
\begin{gathered}
J(x)=\sum_{\alpha=1}^{p} J^{(\alpha)}(x), \\
{\left[J^{(\alpha)}(x), J^{(\beta)}(y)\right]_{q_{\alpha \beta}}=0,}
\end{gathered}
$$

where $q_{\alpha \beta}=1-2 \delta_{\alpha \beta}$. The parabose Grassmann Green components commute with the parabose field Green components for the same value of the Green index and anticommute for different values of the Green index. The external source term $\int d^{4} x(1 / 2)\left(\left[J^{\star}(x), \phi(x)\right]_{+}+\left[J(x), \phi^{\star}(x)\right]_{+}\right)$is then an effective bose operator [15]. Using the fact that

$$
\frac{1}{2}\left[\partial_{\mu} \phi^{\star}(x), \partial^{\mu} \phi(x)\right]_{+}=\sum_{i} \partial_{\mu} \phi^{(\alpha) \star}(x) \partial^{\mu} \phi^{(\alpha)}(x),
$$

we write the generating functional for a free parabose field as

$$
\begin{aligned}
& \int \prod_{\alpha=1}^{p} \mathcal{D} \phi^{(\alpha) \star} \mathcal{D} \phi^{(\alpha)} \exp \left[-i \sum_{1}^{p} \int d^{4} x\left(\partial_{\mu} \phi^{(\alpha) \star}(x) \partial^{\mu} \phi^{(\alpha)}(x)-m^{2} \phi^{(\alpha) \star}(x) \phi^{(\alpha)}(x)\right.\right. \\
+ & \left.\left.\left(J^{(\alpha) \star}(x) \phi^{(\alpha)}(x)+\text { h.c. }\right)\right)\right] .
\end{aligned}
$$


As in the bose case we can do the free path integral to find

$$
W_{\text {free }}\left[J, J^{\star}\right]=W_{\text {free }}[0,0] \exp \left[-i \sum_{\alpha=1}^{p} \int d^{4} x d^{4} y J^{(\alpha) \star}(x) \Delta_{F}(x-y) J^{(\alpha)}(y)\right] .
$$

For interacting theories in which the terms are functions of $\left[\phi^{\star}(x), \phi(x)\right]_{ \pm}$, the interaction term in the Lagrangian is $V_{1}\left(\left[\phi^{\star}(x), \phi(x)\right]_{ \pm}\right)$and we represent the generating functional for the interacting theory as

$$
W\left[J, J^{\star}\right]=\exp \left[i \int d^{4} x V_{1}\left(-\sum_{\alpha=1}^{p} \frac{\delta^{2}}{\delta J^{(\alpha)} \delta J^{(\alpha) \star}}\right)\right] W_{\text {free }}\left[J, J^{\star}\right]
$$

We give just one example of the case where the interaction term in the Lagrangian has the nested commutator form,

$$
\mathcal{L}_{\mathcal{I}}=g \frac{1}{4}\left[\left[\phi_{1}(x), \phi_{2}(x)\right]_{-}, \phi_{3}(x)\right]_{+}=g \sum_{\substack{\alpha, \beta, \gamma \\ \text { all different }}} \phi_{1}^{(\alpha)}(x) \phi_{2}^{(\beta)}(x) \phi_{3}^{(\gamma)}(x)
$$

where $\phi_{i}$ are three different neutral parabose fields of order $p=3$. In this case we need free Lagrangian terms for each of the three fields, so the free generating functional is

$$
W_{\text {free }}\left[J_{i}, J_{j}^{\star}\right]=W_{\text {free }}[0,0] \exp \left[-i \sum_{i=j=1}^{3} \sum_{\alpha=1}^{3} \int d^{4} x d^{4} y J_{i}^{(\alpha \star)}(x) \Delta_{F}(x-y) J_{j}^{(\alpha)}(y)\right]
$$

The interacting generating functional is

$$
W\left[J_{i}, J_{j}\right]=\exp \left[i \int d^{3} x \sum_{\substack{\alpha, \beta, \gamma \\ \text { all different }}} \frac{\delta^{3}}{\delta J_{1}^{(\alpha)} \delta J_{2}^{(\beta)} \delta J_{3}^{(\gamma)}}\right] W_{\text {free }}\left[J_{i}, J_{j}^{\star}\right] .
$$

Note that for this type of interaction the degree of the fields in the interaction Hamiltonian must match the order of the parabose field so that the Green indices are "saturated." In addition, the interacting Hamiltonian should commute with the field. For even degrees the interaction Hamiltonian will anticommute as pointed out 
in [13]. This case should not be allowed because it leads to alternating signs of the contribution of the interaction energy to widely separated states [15].

Now the vacuum matrix elements of the time ordered products of the fields $\phi^{\star}(x)$ and $\phi(x)$ are given by the operations $\sum_{1}^{p} \delta / \delta J^{(\alpha)}(x)$ and $\sum_{1}^{p} \delta / \delta J^{(\alpha) \star}(x)$, respectively, acting on the generating functional. The anticommutativity of the $\phi^{(\alpha)}$ 's and $J^{(\alpha)}$ 's for different values of the Green indices leads to the anticommutativity of the quantized fields for different values of the Green index in the vacuum matrix elements of the time ordered products, and thus leads to the time ordered products of the parabose fields.

The functional differentiation with respect to $J^{(\alpha)}(x)$ can be carried out using the following commutation relation, which is analogous to Eq.(7) for the canonical formalism,

$$
\frac{\delta}{\delta J^{(\alpha)}(x)} J^{(\beta)}(y)= \pm\left(2 \delta_{\alpha, \beta}-1\right) J^{(\beta)}(y) \frac{\delta}{\delta J^{(\alpha)}(x)}+\delta_{\alpha, \beta} \delta(x-y) .
$$

This relation is for a neutral field and the \pm refers to parabose (parafermi). For a charged field, just omit the contractions that do not connect the field with its adjoint.

As specific illustrations, we evaluate the two-point and four-point Green's function for a non-interacting parabose system. We choose to exhibit the results for a neutral field, which has all terms that can occur in the case of a charged field. For a specific distribution of a charged field and its adjoint use the result for the neutral field, but keep only those propagators that connect the charged field and its adjoint. Discard those propagators that would have connected the field with the field or the adjoint with the adjoint.

$$
\begin{aligned}
<0\left|T \phi\left(x_{2}\right) \phi\left(x_{1}\right)\right| 0> & =\left.\sum_{\alpha, \beta=1}^{p}\left(\frac{1}{i}\right)^{2} \frac{\delta^{2}}{\delta J^{(\beta)}\left(x_{2}\right) \delta J^{(\alpha)}\left(x_{1}\right)} W\right|_{J^{(\beta)}=0} \\
& =i p \Delta_{F}\left(x_{1}-x_{2}\right)
\end{aligned}
$$

and

$$
<0\left|T \phi\left(x_{4}\right) \phi\left(x_{3}\right) \phi\left(x_{2}\right) \phi\left(x_{1}\right)\right| 0>=
$$




$$
\begin{aligned}
& \sum_{\alpha, \beta, \gamma, \delta}\left(\frac{1}{i}\right)^{4} \frac{\delta^{4}}{\delta J^{(\delta)}\left(x_{4}\right) \delta J^{(\gamma)}\left(x_{3}\right) \delta J^{(\beta)}\left(x_{2}\right) \delta J^{(\alpha}\left(x_{1}\right)} \times\left. W\right|_{J=0} \\
= & p^{2} i \Delta_{F}\left(x_{1}-x_{2}\right) i \Delta_{F}\left(x_{3}-x_{4}\right)+p(2-p) i \Delta_{F}\left(x_{2}-x_{4}\right) i \Delta_{F}\left(x_{1}-x_{3}\right) \\
& +p^{2} i \Delta_{F}\left(x_{1}-x_{4}\right) i \Delta_{F}\left(x_{2}-x_{3}\right) .
\end{aligned}
$$

In particular, for a charged field, the result is

$$
\begin{aligned}
& <0\left|T \phi^{\star}\left(x_{4}\right) \phi^{\star}\left(x_{3}\right) \phi\left(x_{2}\right) \phi\left(x_{1}\right)\right| 0>= \\
& \sum_{\alpha, \beta, \gamma, \delta}\left(\frac{1}{i}\right)^{4} \frac{\delta^{4}}{\delta J^{(\delta)}\left(x_{4}\right) \delta J^{(\gamma)}\left(x_{3}\right) \delta J^{(\beta) \star}\left(x_{2}\right) \delta J^{(\alpha) \star}\left(x_{1}\right)} \times\left. W\right|_{J, J^{\star}=0} \\
& =p(2-p) i \Delta_{F}\left(x_{1}-x_{3}\right) i \Delta_{F}\left(x_{2}-x_{4}\right)+p^{2} i \Delta_{F}\left(x_{1}-x_{4}\right) i \Delta_{F}\left(x_{2}-x_{3}\right) .
\end{aligned}
$$

The validity of the above path integral based results can be ascertained through the direct evaluation of the time ordered products. In momentum space, the parabose fields are

$$
\begin{gathered}
\phi(x)=\frac{1}{(2 \pi)^{3 / 2}} \int \frac{d^{3} k}{\sqrt{2 \omega_{k}}}\left(b_{k} e^{-i k \cdot x}+d_{k}^{\dagger} e^{i k \cdot x}\right) \equiv \phi^{(+)}(x)+\phi^{(-)}(x), \\
\phi^{\star}(x)=\frac{1}{(2 \pi)^{3 / 2}} \int \frac{d^{3} k}{\sqrt{2 \omega_{k}}}\left(d_{k} e^{-i k \cdot x}+b_{k}^{\dagger} e^{i k \cdot x}\right) \equiv \phi^{\star(+)}(x)+\phi^{\star(-)}(x),
\end{gathered}
$$

and these satisfy the vacuum conditions

$$
\phi^{(+)}(x)\left|0>=\phi^{\star(+)}\right| 0>=0 .
$$

Substitution of Eqs.(30, 31) in the time ordered products, subsequent expansion of the creation and annihilation operators in terms of Green components, and the application of vacuum condition Eq.(32) again lead to the expressions Eq.(26) and Eq.(28) for the two and four point Green's functions.

\subsection{Parafermi case}

Next we consider the path integral for parafermions. For a parafermi field we interchange the roles of commutators and anticommutators relative to the case of 
parabose fields. Thus the Lagrangian density for a free parafermi field is

$$
\begin{aligned}
\mathcal{L}(\psi, \bar{\psi}) & =\frac{1}{2}[\bar{\psi}(x),(i \not \nabla-m) \psi(x)]_{-} \\
& =\sum_{\alpha=1}^{p}\left(\bar{\psi}^{(\alpha)}(x)(i \not \nabla-m) \psi^{(\alpha)}(x)\right)+\mathrm{c}-\text { number } .
\end{aligned}
$$

The generating functional for a free charged parafermi field is

$$
\begin{aligned}
& \int \prod_{\alpha=1}^{p} \mathcal{D} \bar{\psi}^{(\alpha) \star} \mathcal{D} \psi^{(\alpha)} \exp \left[-i \sum_{1}^{p} \int d^{4} x\left(\bar{\psi}^{(\alpha)}(x)(i \not \nabla-m) \psi^{(\alpha)}(x)\right.\right. \\
& \left.\left.+\bar{\eta}^{(\alpha)}(x) \psi^{(\alpha)}(x)+\bar{\psi}^{(\alpha)}(x) \eta^{(\alpha)}(x)\right)\right] .
\end{aligned}
$$

Now we replace the path integral over commuting fields by the Berezin path integral [16] over Grassmann fields and require the Grassmann fields $\bar{\psi}^{(\alpha)}(x)$ and $\psi^{(\alpha)}(x)$ and the external sources $\bar{\eta}^{(\alpha)}(x)$ and $\eta^{(\alpha)}(x)$ to anticommute for the same and commute for different values of the Green indices. The generating functional for a free parafermi field is

$$
W_{\text {free }}[\eta, \bar{\eta}]=W_{\text {free }}[0,0] \exp \left[-i \sum_{\alpha=1}^{p} \int d^{4} x d^{4} y \bar{\eta}^{(\alpha)}(x) S_{F}(x-y) \eta^{(\alpha)}(y)\right]
$$

where $S_{F}(x)=(2 \pi)^{-4} \int d^{4} p \exp (-i p \cdot x)(\not p+m)\left(p^{2}-m^{2}+i \epsilon\right)^{-1}$. In the presence of an interaction $V(\bar{\psi}, \psi)$, we modify the generating functional to

$$
W[\eta, \bar{\eta}]=\exp \left[i \int d^{4} x V\left(-i \frac{\delta}{\delta \eta(x)},-i \frac{\delta}{\delta \bar{\eta}(x)}\right)\right] W_{\text {free }}[\eta, \bar{\eta}]
$$

These arguments can be extended further to systems in which parafermi and parabose fields have mutual interaction. As a specific example of such systems, we consider the Yukawa coupling between a charged parafermi and a neutral parabose fields of order 3, respectively [13]. These fields obey relative para-Bose commutation rules. The interaction term is

$$
\begin{aligned}
\mathcal{L}_{\mathcal{I}} & =g \frac{1}{4}\left[[\bar{\psi}(x), \psi(x)]_{+}-<[\bar{\psi}(x), \psi(x)]_{+}>_{o}, \phi(x)\right]_{+} \\
& =g \sum_{\substack{\alpha, \beta, \gamma \\
\text { all different }}} \bar{\psi}^{(\alpha)}(x) \psi^{(\beta)}(x) \phi^{(\gamma)}(x)
\end{aligned}
$$


The generating functional for the noninteracting system is now

$$
\begin{aligned}
& W_{\text {free }}[\eta, \bar{\eta}, J]= \\
& W_{\text {free }}[0,0,0] \exp \left[-i \sum_{\alpha=1}^{3} \int d^{4} x d^{4} y \bar{\eta}^{(\alpha)}(x) S_{F}(x-y) \eta^{(\alpha)}(y)\right] \\
& \times \exp \left[-i \sum_{\gamma=1}^{3} \int d^{4} x d^{4} y J^{(\gamma)}(x) \Delta_{F}(x-y) J^{(\gamma)}(y)\right]
\end{aligned}
$$

which leads to the following expression for the complete generating functional

$$
W[\eta, \bar{\eta}, J]=\exp \left[-\int d^{4} x \sum_{\begin{array}{c}
\alpha, \beta, \gamma \\
\text { all different }
\end{array}} \frac{\delta^{3}}{\eta^{(\alpha)}(x) \bar{\eta}^{(\beta)}(x) J^{(\gamma)}(x)}\right] W_{\text {free }}[\eta, \bar{\eta}, J] .
$$

The propagators for the parafermi system can be obtained by employing the commutation relation Eq.(26) with the lower sign. Note that proceeding as in the parabose case, we get, for example, the following expression for a four-point parafermi propagator

$$
\begin{aligned}
& <0\left|T \psi\left(x_{4}\right) \psi\left(x_{3}\right) \bar{\psi}\left(x_{2}\right) \bar{\psi}\left(x_{1}\right)\right| 0>= \\
& \left.\sum_{i_{1}, i_{2}, i_{3}, i_{4}} i^{4} \frac{\delta^{4}}{\delta \bar{\eta}^{\left(i_{4}\right)}\left(x_{4}\right) \delta \bar{\eta}^{\left(i_{3}\right)}\left(x_{3}\right) \delta \eta^{\left(i_{2}\right)}\left(x_{2}\right) \delta \eta^{\left(i_{1}\right)}\left(x_{1}\right)} W\right|_{\eta=\bar{\eta}=0} \\
& =p^{2} i S_{F}\left(x_{3}-x_{2}\right) i S_{F}\left(x_{4}-x_{1}\right)-p(2-p) i S_{F}\left(x_{3}-x_{1}\right) i S_{F}\left(x_{4}-x_{2}\right) .
\end{aligned}
$$

One can construct all other generating functionals for para fields, such as the generating functionals for connected and for one-particle-irreducible Greens functions, in parallel with usual cases, just as we have done above for the time-ordered products.

\section{Remark about the partition function}

The single-particle propagator is related to the partition function [17]. Our result Eq.(28) for the two-particle propagator gives a result for the partition function that 
is the square of the partition function in the special case $p=2$ as we expect from the result of $\mathrm{P}$. Suranyi [18] that a gas of $p=2$ parabosons (or parafermions) is equivalent to a mixture of two independent gases of bosons (or fermions). We thank A. Polychronakos [19] for pointing out that the different four-point matrix element that we gave in an earlier version of this work

$$
\begin{aligned}
& <0\left|T \phi^{\star}\left(x_{4}\right) \phi\left(x_{3}\right) \phi^{\star}\left(x_{2}\right) \phi\left(x_{1}\right)\right| 0>= \\
& \sum_{\alpha, \beta, \gamma, \delta}\left(\frac{1}{i}\right)^{4} \frac{\delta^{4}}{\delta J^{\delta)}\left(x_{4}\right) \delta J^{\left(i_{3}\right) \star}\left(x_{3}\right) \delta J^{\left(i_{2}\right)}\left(x_{2}\right) \delta J^{\left(i_{1}\right) \star}\left(x_{1}\right)} \times\left. W\right|_{J=0} \\
& =p^{2}\left[i \Delta_{F}\left(x_{1}-x_{2}\right) i \Delta_{F}\left(x_{3}-x_{4}\right)+i \Delta_{F}\left(x_{1}-x_{4}\right) i \Delta_{F}\left(x_{2}-x_{3}\right)\right] .
\end{aligned}
$$

does not have this property.

\section{Remark about normalization of para operators}

We have followed the normalization used by Green [9] when he introduced parastatistics in which the parameter $p$ that labels the order of the parastatistics does not appear in the commutation relations Eq.(3), but $p$ does appear in the normalization of the single-particle state Eq.(5). Green's motivation was to keep the usual commutation relations between the number operator and the annihilation and creation operators Eq.(1). One can normalize the single-particle state to one, but then the commutation relation becomes

$$
\left[\left[b_{k}^{\dagger}, b_{l}\right]_{ \pm}, b_{m}^{\dagger}\right]_{-}=2 p \delta_{l m} b_{k}^{\dagger}
$$

and the number operator commutation relations also acquire the factor $p$.

\section{Comparison with earlier work}

We are aware of three earlier treatments of parastatistics using path integrals. Y. Ohnuki and S.Kamefuchi [20] construct the path integral for parafermi fields using paragrassmann variables following the fundamental definition of a path integral as the limit of a product of time evolution operators for small time intervals. They 
do not evaluate this limit and thus do not find an explicit formula for the path integral. They also do not give the generating functions that are most useful for calculations. Like our construction, their analysis relies on the Green ansatz and does not give an intrinsic construction of the path integral. M. Chaichian and A. Demichev [21] and A. Polychronakos [22] both give an intrinsic definition of the path integral that does not rely on the Green ansatz. Their construction of the path integral is a first quantized analog of using the Green trilinear commutation relations without making use of the Green ansatz. They define the propagator for paraparticles using the propagator for distinguishable particles together with the matrix elements of the permutation operator. They point out that these matrix elements are "weights for each topological sector of the path integral" that are simply positive or negative integers or zero. The result of Polychronakos that, in his notation, $S_{p} F(P)=(-1)^{P} S_{p} B(P)$, follows in our case from the \pm sign in Eq. $(7,26)$.

\section{Summary and conclusions}

We have shown that parastatistics fields can be quantized using path integrals. All the results are, as we expect, the same as found with the canonical formalism. Up to now we have not found a computational advantage in using either of these formalism for parastatistics. We explicitly calculated the two- and four-point functions for free parabose and free parafermi theories and also gave the generating functionals for both free and interacting theories. It would be interesting to formulate the path integral for parastatistics fields using only a definition of the path integral that uses the trilinear commutation relations for parafields without reference to Green's ansatz.

\section{Acknowledgements}

We are happy to acknowledge the suggestion of John Tjon that stimulated us to write this paper. We also greatly appreciate the suggestions of John Tjon, Masud Chaichian and Alexios Polychronakos that significantly improved our paper. We thank Cecile DeWitt for clarifying the work of Laidlaw and DeWitt [5]. The work 
of OWG was supported in part by National Science Foundation Grant No. PHY0140301 and in part by National Science Foundation Award No. INT-0223818. The work of AKM was supported in part by National Science Foundation Award No. INT-0223818 and by the Department of Science and Technology of India.

\section{References}

[1] W. Heisenberg and W. Pauli, Z. Physik 56, 1 (1929).

[2] S. Weinberg, The Quantum Theory of Fields, Vol I, (Cambridge, New York, 1995).

[3] R.P. Feynman. Rev. Mod. Phys. 20, 367 (1948).

[4] M. Chaichian and A. Demichev, Path Integrals in Physics, Vol. I, II, (Institute of Physics, Bristol, 2001).

[5] M.G.G. Laidlaw and C.M. DeWitt, Phys. Rev. D3, 1375 (1971).

[6] J.M. Leinaas and J. Myrheim, Nuovo Cimento 37, 1 (1977).

[7] F. Wilczek, Fractional Statistics and Anyon Superconductivity, (World, Singapore, 1990).

[8] Cecile DeWitt, private communication.

[9] H.S. Green, Phys. Rev. 90, 270 (1953).

[10] A.M.L. Messiah and O.W. Greenberg, Phys. Rev. 136, B248 (1964).

[11] R.C. Hilborn and C.L. Yuca, British J. Phil. Science 53, 355 (2002) gives later references.

[12] R. D. Amado and H. Primakoff, Phys. Rev. C 22, 1338 (1980).

[13] O.W. Greenberg and A.M.L. Messiah, Phys. Rev. 138, B1155 (1965).

[14] H. Araki, O.W. Greenberg and J.S. Toll, Phys. Rev. 142, 1017 (1966). 
[15] O.W. Greenberg, Phys. Lett. A 209, 137 (1995).

[16] F.A. Berezin, The Method of Second Quantization, (Academic Press, New York, 1966).

[17] L.S. Schulman, Techniques and Applications of Path Integration, (WileyInterscience, New York, 1981).

[18] P. Suranyi, Phys. Rev. Lett. 65, 2329 (1990).

[19] A. Polychronakos, private communication, (2004).

[20] Y. Ohnuki and S.Kamefuchi, Quantum Field Theory and Parastatistics, (University of Tokyo, Tokyo,1982), pp406-408.

[21] M. Chaichian and A. Demichev, Path Integrals in Physics, Vol. II, (Institute of Physics, Bristol, 2001), pp216-221.

[22] A.P. Polychronakos, Nucl. Phys. B474, 529 (1996). 